\title{
Discrepancies in the measurement of isovolumic relaxation time: a study comparing $M$ mode and Doppler echocardiography
}

\author{
C H Lee, F Vancheri, M S Josen, D G Gibson
}

\begin{abstract}
Mitral valve cusp separation on $M$ mode echogram, the mitral valve opening artefact, and the onset of forward transmitral flow recorded by Doppler echocardiography have all been taken to mark the end of isovolumic relaxation, while its onset has been taken either as the aortic closure sound $\left(A_{2}\right)$ recorded phonocardiographically or the aortic closure artefact determined by Doppler technique. Possible differences in the measurement of the isovolumic relaxation time were studied when these landmarks were used in 44 healthy people, 14 patients with mitral stenosis, 21 patients with left ventricular hypertrophy, and 24 patients with dilated cardiomyopathy by recording $M$ mode echograms of the mitral valve, and pulsed and continuous wave Doppler spectra of transmitral flow, with simultaneous electrocardiograms and phonocardiograms. $A_{2}$ was effectively synchronous with the aortic artefact. However, when the onset of Doppler flow was regarded as the end of isovolumic relaxation, the interval was significantly longer than when mitral cusp separation on $M$ mode echograms was used: by 25 (10) $\mathrm{ms}$ in healthy individuals, by 25 (15) $\mathrm{ms}$ in patients with left ventricular hypertrophy, and by 50 (35) ms in patients with dilated cardiomyopathy. In patients with mitral stenosis the interval was only $5(5) \mathrm{ms}$ longer. The mitral valve opening artefact consistently followed the onset of flow and corresponded much more closely to the $E$ point on the $M$ mode echogram. This shows that it occurred during the rapid filling period and well beyond isovolumic relaxation by any definition.
\end{abstract}

Thus isovolumic relaxation time measured from $A_{2}$ to the onset of transmitral flow or the mitral valve opening artefact differs from that derived from $A_{2}$ to mitral valve cusp separation. These intervals cannot be used interchangeably to measure "isovolumic relaxation time".

Isovolumic relaxation is an important phase of the cardiac cycle. Its length has been used to assess left ventricular diastolic function, ${ }^{1}$ to distinguish myocardial restriction from constriction, ${ }^{2}$ and to assess the cardiac effects of pharmacological interventions. ${ }^{3}$ In his classic text Wiggers wrote of this interval (initially termed isometric relaxation): "Following the closure of the semilunar valves, and until the $A-V$ valves have opened, the ventricle relaxes without any flow of blood either from or into its cavity. This phase may be designated as the isometric relaxation phase". ${ }^{4}$ Isovolumic relaxation time has been measured on simultaneous $M$ mode and phonocardiogram recordings as the interval from aortic valve closure $\left(\mathrm{A}_{2}\right)$ to the point of mitral cusp separation. More recently, the determination of isovolumic relaxation time has formed part of the Doppler evaluation of left ventricular diastolic function where it has been taken variously as the interval from $A_{2}$ to the onset of transmitral flow, $A_{2}$ to the mitral valve opening artefact on pulsed wave Doppler recordings, or the aortic valve closure artefact to the onset of transmitral flow recorded by continuous wave Doppler. ${ }^{5}$ It has been implicitly assumed that all these measurements are equivalent. Mitral cusp separation is a complex phenomenon, however, and is influenced by many factors apart from transmitral flow, so these different measurements need not necessarily be identical. We therefore explored this possibility, aiming not only to resolve possible confusion but also to use these interrelations to shed light on early diastolic events in normal and abnormal hearts.

\section{Patients and methods}

STUDY POPULATION

We studied 44 healthy individuals ( 27 men, 17 women; aged 18-60), 14 patients with mitral stenosis (three men, 11 women; aged 16-75), 21 patients with left ventricular hypertrophy (11 aortic valve disease, five hypertrophic cardiomyopathy, five hypertensive; 11 men, 10 women; aged 23-75), and 24 patients with dilated cardiomyopathy (16 male, 8 female; aged 12-77, 22 of whom had functional mitral regurgitation). All the patients were in sinus rhythm except for 11 patients with mitral stenosis. There were no significant differences in age among the groups studied. 
Figure 1 M mode echogram and phonocardiogram from a patient with dilated cardiomyopathy.

Isovolumic relaxation time, measured as the interval from $A$, to mitral valve opening, is zero. The second heart sound is reversed in this patient because of left bundle branch block.
||||||||||||||||||||||||||||||||||||||||||||||||||||||||||||||||||||||||||||||||||

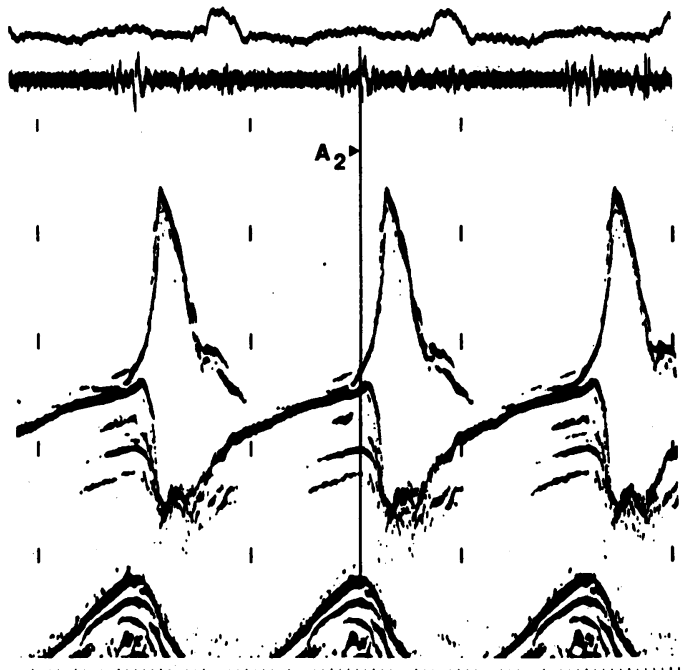

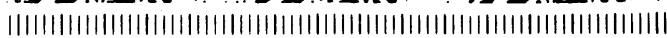

M MODE ECHOCARDIOGRAPHY

We recorded $M$ mode echocardiograms of the mitral valve with a $3.0 \mathrm{MHz}$ transducer and ATL Imager Mk300I equipment in the parasternal long axis view with the patients lying in the left lateral position. The minimum gain setting was used to show both the anterior and posterior mitral cusps. We regarded the point of cusp separation as the time of mitral valve opening; cusp separation was clearly defined on all records (fig 1 ). We did not use $\mathrm{D}$ or $\mathrm{D}^{\prime}$ points because they were poorly defined and thus unreproducible. The $E$ point of the mitral echogram was taken as the point of maximum anterior cusp motion in early diastole. Phonocardiograms and electrocardiograms were recorded simultaneously with a Honeywell recorder at a paper speed of $10 \mathrm{~cm} / \mathrm{s}$.

\section{DOPPLER ECHOCARDIOGRAPHY}

Doppler recordings were made with Doptek equipment and a $2 \cdot 25 \mathrm{MHz}$ transducer. Forward transmitral flow was recorded in all patients. Peak mitral flow velocity was initially identified by continuous wave Doppler and then recorded with the pulsed mode and a 3 $\mathrm{mm}$ gate. A wall filter of $250 \mathrm{~Hz}$ was used in all cases. During the recordings we used the minimum gain setting that gave a complete envelope. The onset of transmitral flow was taken as the time at which the signal rose above the wall filter and was routinely distinguished from the valve artefact, which was recorded separately. Functional mitral regurgitation was carefully sought in all patients by continuous wave Doppler echocardiography. Simultaneous phonocardiograms and electrocardiograms were again recorded at a paper speed of $10 \mathrm{~cm} / \mathrm{s}$. We used continuous wave Doppler echocardiography directed towards the left ventricular outflow tract to obtain recordings showing both the aortic valve closure artefact and the transmitral flow.

\section{MEASUREMENTS}

Aortic valve closure $\left(A_{2}\right)$ was taken as the onset of the first high frequency component of the second sound recorded on the phonocardiogram, which was routinely checked against the closure point on the aortic echogram. The following measurements were made on the $M$ mode recordings (fig 1): (a) The interval from $A_{2}$ to mitral cusp separation; (b) the interval from $A_{2}$ to the $E$ point; (c) the interval from minimum left ventricular dimension to mitral cusp separation-when this was $>30 \mathrm{~ms}$ it was regarded as indicating incoordinate relaxation. ${ }^{6}$ We measured the following intervals on the pulsed wave Doppler recordings (figs $2 A$ and $B$ ): (a) from $A_{2}$ to the onset of transmitral flow; $(b)$ from $A_{2}$ to the leading edge of mitral valve opening artefact; and (c) from $A_{2}$ to the peak transmitral flow velocity. The interval from the leading edge of the aortic valve closure artefact to the onset of transmitral flow was measured on the continuous wave Doppler recordings (fig 3). We took the mean of measurements made on three cardiac cycles.

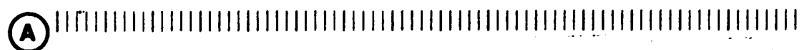
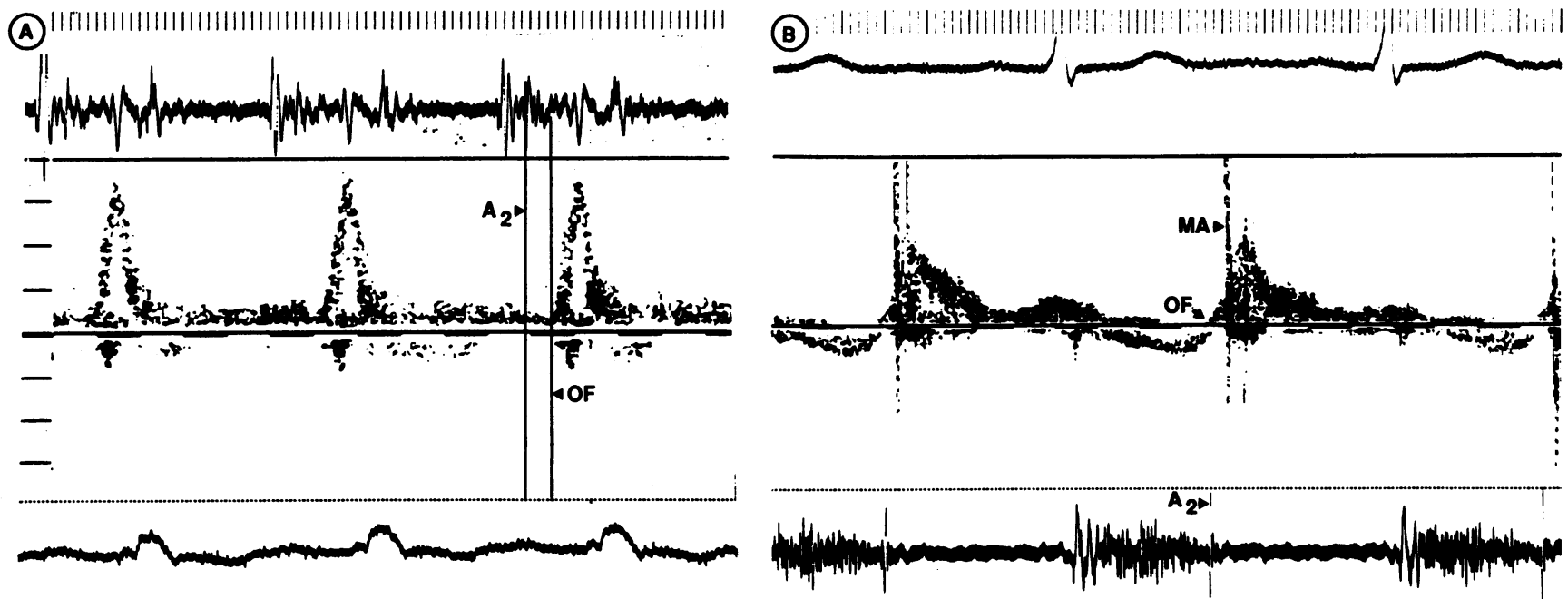

Figure 2 (A) Pulsed Doppler echocardiogram from the same patient as figure 1 showing an interval of $40 \mathrm{~ms}$ from $\mathrm{A}_{2}$ to the onset of forward transmitral flow (OF). Full scale deflection $=4 \mathrm{kHz}$. (B) Pulsed Doppler echocardiogram showing the relation between $A_{2}$, the onset of forward transmitral flow (OF), and the mitral valve opening artefact (MA). Note that the mitral valve opening artefact consistently follows the onset of flow. Full scale deflection $=8 \mathrm{kHz}$. 


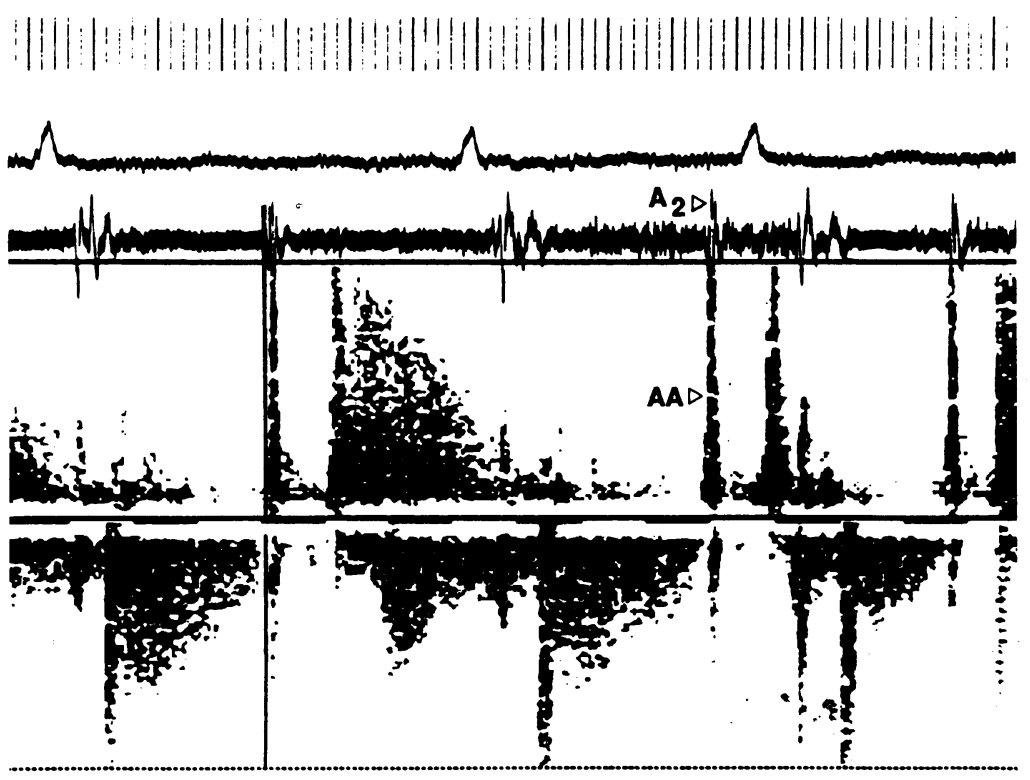

Figure 3 Continuous wave Doppler echocardiogram from a patient with mitral stenosis and atrial fibrillation showing both the aortic valve closure artefact $(A A)$ and the onset of transmitral flow. The leading edge of the aortic closure artefact is synchronous with the onset of the first high frequency deflection of the second sound $\left(A_{2}\right)$. Full scale deflection $=4 \mathrm{kHz}$.

\section{Results}

ISOVOLUMIC RELAXATION TIME (TABLE 1 AND TABLE 2)

Healthy individuals

The interval between $A_{2}$ and mitral cusp separation was consistently less than that between $\mathrm{A}_{2}$ and the onset of flow. The interval from cusp separation to the earliest detectable mitral flow was consistently greater than zero (25 (10) ms) ( $\mathrm{p}<0.0001)$. The mitral valve artefact consistently followed (by $25(10) \mathrm{ms}$ ) the onset of flow $(p<0.0001)$. The leading edge of the aortic closure artefact was synchronous with the onset of the first high frequency deflection of the second sound recorded on the phonocardiogram and the interval between this artefact and the onset of flow on continuous wave Doppler was identical to that determined by pulsed mode.

\section{Left ventricular hypertrophy}

The mean values for the $M$ mode and Doppler measurements were similar to those of healthy individuals. The scatter about the mean, however, was much greater. In 11 patients with coordinate relaxation (minimum left ventricular dimension to mitral valve opening $<30$ $\mathrm{ms}$ ) the difference between the onset of transmitral flow and mitral cusp separation was 15 (10) ms, while in 10 patients with incoordinate relaxation (minimum cavity dimension to mitral valve opening interval $>30 \mathrm{~ms}$ ) the difference was larger (40 (11) ms).

\section{DILATED CARDIOMYOPATHY}

This group of patients had the shortest interval between $\mathrm{A}_{2}$ and mitral cusp separation. The interval between $A_{2}$ and the onset of flow, though shorter than that in healthy individuals, was much longer than the interval between $A_{2}$ and mitral cusp separation. There was a delay of 50 (35) ms between mitral cusp separation and the onset of transmitral flow.

\section{MITRAL STENOSIS}

The $M$ mode and Doppler measurements of isovolumic relaxation time were all shorter than those of healthy individuals. There was no

Table 1 Measurements (mean (SD)) of isovolumic relaxation time

\begin{tabular}{lllll}
\hline Group & $\begin{array}{l}A_{2} \text { to mitral cusp } \\
\text { separation (ms) }\end{array}$ & $\begin{array}{l}A_{2} \text { to onset of } \\
\text { flow (ms) }\end{array}$ & $\begin{array}{l}A_{2} \text { to mitral valve } \\
\text { artefact (ms) }\end{array}$ & $\begin{array}{l}\text { Aortic valve artefact to } \\
\text { onset of flow (ms) }\end{array}$ \\
\hline Healthy individuals & $60(15)$ & $85(15)$ & $115(25)$ & $85(25)$ \\
Left ventricular hypertrophy & $65(25)$ & $90(30)$ & $110(25)$ & $90(15)$ \\
Dilated cardiomyopathy & $15(30)$ & $65(40)$ & $90(25)$ & $65(25)$ \\
Mitral stenosis & $50(25)$ & $55(30)$ & $80(30)$ & $55(20)$ \\
\hline
\end{tabular}

Table 2 Differences between measurements

\begin{tabular}{|c|c|c|c|c|c|c|}
\hline \multirow[b]{2}{*}{ Group } & \multicolumn{3}{|c|}{$\begin{array}{l}A_{2} \text { to onset of flow and } \\
A_{2} \text { to mitral cusp separation }\end{array}$} & \multicolumn{3}{|c|}{$\begin{array}{l}A_{2} \text { to mitral valve artefact and } \\
A_{2} \text { to onset of flow }\end{array}$} \\
\hline & $\begin{array}{l}\text { Difference } \\
\text { (ms) }\end{array}$ & pvalue & $\begin{array}{l}95^{\circ} \text { o confidence } \\
\text { interval }(\mathrm{ms})\end{array}$ & $\begin{array}{l}\text { Difference } \\
\text { (ms) }\end{array}$ & pvalue & $\begin{array}{l}95 \% \text { confidence } \\
\text { interval (ms) }\end{array}$ \\
\hline $\begin{array}{l}\text { Healthy individuals } \\
\text { Left ventricular hypertrophy } \\
\text { Dilated cardiomyopathy } \\
\text { Mitral stenosis }\end{array}$ & $\begin{array}{c}25(10) \\
25(15) \\
50(35) \\
5(5)\end{array}$ & $\begin{array}{l}<0.0001 \\
<0.005 \\
<0.0001 \\
>0.05\end{array}$ & $\begin{array}{r}19-33 \\
8-40 \\
32-72 \\
-14-27\end{array}$ & $\begin{array}{l}25(10) \\
20(20) \\
15(10) \\
15(5)\end{array}$ & $\begin{array}{l}<0.0001 \\
<0.01 \\
<0.05 \\
<0.05\end{array}$ & $\begin{array}{r}15-40 \\
5-40 \\
10-35 \\
-14-53\end{array}$ \\
\hline
\end{tabular}

$P$ values show significance of differences between measured intervals and zero. 
Table 3 Coefficients of correlation

\begin{tabular}{lllr}
\hline Group & Flow delay $v$ & Flow delay $v$ \\
A to mitral cusp separation & Flow delay v age & RR interval \\
\hline Healthy individuals & -0.221 & 0.132 & -0.091 \\
Left ventricular hypertrophy & 0.016 & $0.453^{\star}$ & $0.453^{\star}$ \\
Dilated cardiomyopathy & -0.271 & 0.227 & -0.028 \\
Mitral stenosis & 0.020 & $-0.539^{\star}$ & -0.007
\end{tabular}

Flow delay = interval from $A_{2}$ to onset of flow minus interval from $A_{2}$ to mitral cusp separation.

$\star_{\mathrm{p}}<0.05$.

significant delay in the onset of transmitral flow after mitral cusp separation (5 (5) $\mathrm{ms}$ ). The mitral valve artefact also followed the onset of flow in these patients but the difference was not significant.

\section{CORRELATIONS (TABLE 3 )}

The delay in the onset of transmitral flow after mitral cusp separation showed no significant correlation with the interval between $A_{2}$ and mitral cusp separation. A weak correlation was seen between the delay in flow and age in patients with left ventricular hypertrophy or mitral stenosis, and between the delay in flow and $R R$ interval in patients with left ventricular hypertrophy.

\section{RELATION BETWEEN MITRAL VALVE ARTEFACT, MITRAL E POINT, AND DOPPLER PEAK FLOW (TABLE 4)}

The mitral valve artefact did not correspond with the mitral cusp separation, which occurred before the onset of flow. Instead, the mitral valve artefact coincided closely with the $E$ point of the mitral echogram, both in healthy individuals and in the patients. The peak transmitral flow velocity detected on Doppler occurred about $60 \mathrm{~ms}$ after the valve was fully opened, while the mitral valve was actually closing.

\section{Discussion}

When he originally defined isometric relaxation time, Wiggers regarded mitral valve opening and the onset of transmitral flow as synonymous. This assumption was not affected when the name was later changed to isovolumic relaxation time. More recently, many others, including us, ${ }^{6}$ have also assumed that the time from $A_{2}$ to mitral valve opening measures the isovolumic relaxation time unambigously. But this is not so. Several experimental studies showed significant discrepancies between mitral valve motion and transmitral flow. Tsakiris et al marked the anterior mitral valve leaflet of dogs with radio-opaque clips and correlated motion of the marker with simultaneous high fidelity intracardiac pressure monitoring. ${ }^{7}$ They showed that the point of mitral cusp separation preceded the diastolic pressure crossover (when ventricular pressure fell below atrial pressure) and thus the onset of flow by $5-60 \mathrm{~ms}^{7}$ Pohost et al also saw this delay. ${ }^{8}$ In dogs with radio-opaque markers on the mitral valve to show leaflet motion, contrast medium injected into the left atrium flowed beyond the free edges of the mitral valve 43 (3) ms after the initial separation of anterior and posterior leaflets and it did not begin to mix with left ventricular blood until the valve was almost fully open. Estimates of isovolumic relaxation in man made angiographically, when "mitral valve opening" was marked by the first appearance within the ventricular cavity of unopacified blood from the left atrium, again gave normal values that were consistently longer than those measured in the same laboratory by $M$ mode echocardiography. ${ }^{9} \mathrm{~A}$ previous study in healthy individuals by a technique similar to ours showed some delay in Doppler flow after mitral valve opening, although the difference was much smaller (5 $\mathrm{ms}) .{ }^{10}$ Although the mechanisms underlying mitral cusp separation are not clear, ${ }^{11}$ mitral valve opening must thus be regarded as potentially separate from the onset of transmitral flow.

Until recently, isovolumic relaxation time was measured by $M$ mode echocardiography. Now it is increasingly determined by Doppler echocardiography. The details of the technique were often not specified but seem to have used either the onset of forward transmitral flow or the mitral valve opening artefact ${ }^{12}$ as the landmark for mitral valve opening. These measurements have all been called the isovolumic relaxation time, which implies that they are all equivalent. Our results, however, show that both in healthy individuals and in patients with heart disease, mitral cusp separation consistently precedes the onset of flow, which itself precedes the appearance of the mitral valve opening artefact. Indeed the mitral valve artefact coincides closely with the $E$ point of the mitral echogram and thus the fully open position of the valve. While the intervals between $A_{2}$ and mitral cusp separation and $A_{2}$ and onset of flow, though consistently different, do fall within Wiggers's original definition of

Table 4 Relation between valve artefact, mitral E point, and Doppler peak flow

\begin{tabular}{llll}
\hline Group & $A_{2}$ to mitral E point (ms) & $A_{2}$ to mitral artefact (ms) & $A_{2}$ to Doppler peak flowv (ms) \\
\hline Healthy individuals & $130(20)$ & $115(25)$ & $180(25)$ \\
Left ventricular hypertrophy & $120(30)$ & $110(25)$ & $170(35)$ \\
Dilated cardiomyopathy & $90(30)$ & $90(25)$ & $140(45)$ \\
Mitral stenosis & $90(25)$ & $80(30)$ & Not available \\
\hline
\end{tabular}


isovolumic relaxation, the interval between $A_{2}$ and the mitral valve artefact does not, because it includes a significant portion of the rapid filling period.

Spectral analysis of Doppler signals introduces potential errors in the timing of flow. Analysis of very low frequency shifts takes a finite interval that may introduce error. We therefore used a lower filter of $250 \mathrm{~Hz}$ throughout our study and took the onset of flow as the first recordable signal above this level. Extrapolation of the spectral display to the zero flow baseline, which assumed constant acceleration, suggested that any delay introduced by this method cannot be more than $5 \mathrm{~ms}$. The lack of significant delay in signal analysis is also supported by the observation that in patients with mitral stenosis, the onset of flow was effectively synchronous with mitral valve opening. The onset of transmitral flow recorded by the pulsed mode also coincided with that determined by continuous wave Doppler echocardiography, indicating that the timing of the onset of flow was not affected by the position of the sample volume. We conclude therefore that technical factors cannot explain our observations; mitral cusp separation, the onset of transmitral flow, and mitral valve artefact follow one another in an ordered sequence and cannot be regarded as synchronous.

In the group with left ventricular hypertrophy the mean values of isovolumic relaxation time and the delay in flow were the same as in the healthy individuals, although the scatter was greater. When relaxation was incoordinate, however, there was a much longer delay in the onset of flow after mitral cusp separation. The delay in the onset of flow may thus be an indicator of this aspect of diastolic dysfunction. The patients with dilated cardiomyopathy would be expected to have high left atrial pressure, and thus a short isovolumic relaxation time. ${ }^{13}$ This was indeed the case; these patients had short intervals between $A_{2}$ and mitral cusp separation and between $A_{2}$ and onset of flow. There was, however, a large difference between the two measurements, probably because 22 of the 24 patients had functional mitral regurgitation. This persisted for more than $50 \mathrm{~ms}$ beyond $\mathrm{A}_{2}$. Because forward flow cannot occur during regurgitation, the onset of transmitral forward flow is considerably delayed. ${ }^{14}$

As well as simply dealing with the methods of measuring isovolumic relaxation time, our results may be of more fundamental pathophysiological importance. We have previously suggested that restricted left ventricular filling time may be an important cause of diastolic dysfunction and its increase with the fall in heart rate may account for the therapeutic effect of $\beta$ blockade in some patients with severe left ventricular disease and functional mitral regurgitation. ${ }^{14}$ It now seems that the onset of transmitral flow may be delayed after mitral valve opening not only in patients with functional mitral regurgitation but also in those without, especially when relaxation is incoordinate. This delay may well restrict left ventricular filling time when heart rate is increased with exercise, causing stroke volume to be limited by a purely diastolic mechanism that is independent of any systolic disease that may be present.

The term "isovolumic relaxation time", even in terms of the original definition of Wiggers, is ambiguous. Details of measurement must be given in full, since not only do the various methods give results that are numerically different, but they are quite distinct in their pathophysiological significance. The delay in the onset of flow after mitral cusp separation, although predictable in healthy individuals, may vary widely in disease and may come to occupy a considerable proportion of the potential filling time. It may thus be a potentially major cause of diastolic dysfunction when the heart rate is rapid. The $M$ mode and Doppler derived measurements should thus be regarded as complementary and be studied together, rather than separately, to increase our understanding of these complex early diastolic events.

1 Nishimura RA, Housmans PR, Hatle LK, Tajik AJ. Assessment of diastolic function of the heart: background and current applications of Doppler echocardiography. Part 1. Physiologic and pathophysiologic features. Mayo Clin Proc 1989;64:71-81.

2 Hatle LK, Appleton CP, Popp RL. Differentiation of constrictive pericarditis and restrictive cardiomyopathy by Doppler echocardiography. Circulation 1989;79. 357-70.

3 Hanrath P, Mathey DG, Kremer P, Sonntag F, Bleifeld W. Effect of verapamil on left ventricular isovolumic relaxation time and regional left ventricular filling in hypertrophic cardiomyopathy. Am J Cardiol 1980;45:1258-64.

4 Wiggers CJ. The dynamics of the heart beat. In: Modern aspect of the circulation in health and disease. Philadelphia and New York: Lea and Febiger, 1923:99.

5 Sasson Z, Hatle LK, Appleton CP, Jewett M, Alderman EL, Popp RL. Intraventricular flow during isovolumic relaxation: description and characterization by Doppler ech. description and characterization by Doppler

6 Chen W Gion ventricular wall movement in man. Br Heart J 1979; 42:51-6.

7 Tsakiris AG, Gordon DA, Padiyar R, Frechette D. Relation of mitral valve opening and closure to left atrial and ventricular pressures in the intact dog. Am J Physiol 978;234:

8 Pohost GM, Dinsmore RE, Rubenstein JJ, et al. The echocardiogram of the anterior leaflet of the mitral valve. Correlation with hemodynamic studies in dogs. Circulation 1975;51:88-97.

9 Gibson DG, Prewitt TA, Brown DJ. Analysis of left ventricular wall movement during isovolumic relaxation and its relation to coronary artery disease. Br Heart 1976;38:1010-9.

10 Shapiro LM, Thwaites BC. Measurement of isovolumic relaxation: comparison of echocardiographic mitral valve opening and Doppler mitral valve flow. Cardiovasc Res 1987;21:489-91.

11 Brockman SK. Mechanism of the movements of the atrioventricular valves. Am J Cardiol 1966;17:682-90.

12 Klein AL, Hatle LK, Burstow DJ, et al. Doppler characterisation of left ventricular diastolic function in cardiac isation of left ventricular diastolic function in
amyloidosis. $J \mathrm{Am}$ Coll Cardiol 1989;13:1017-26.

13 Mattheos M, Shapiro E, Oldershaw PJ, Sacchetti R, Gibson DG. Non-invasive assessment of changes in left venDG. Non-invasive assessment of changes in left ven-
tricular relaxation by combined phono-, echo-, and tricular relaxation by combined phono-, echo-,
mechanocardiography. Br Heart $J$ 1982;47:253-60.

$14 \mathrm{Ng} \mathrm{KSK}$, Gibson DG. Impairment of diastolic function by shortened filling period in severe left ventricular disease. Br Heart J 1989;62:246-52. 